Pacific Journal of Mathematics

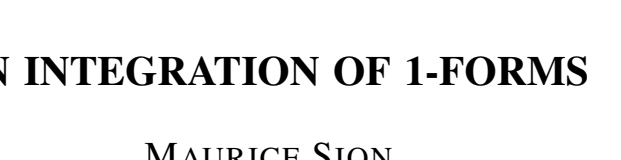




\section{ON INTEGRATION OF 1-FORMS}

\section{MAURICE Sion}

1. Introduction. It has been noted by several people that in order to define the integral of some differential 1-form $\omega$ along a curve $C$, the latter need not be of bounded variation. For example, in the extreme (and trivial) case where $\omega$ is the differential of some function $f$, the integral can be defined as the difference of the values assumed by $f$ at the end-points of $C$. No condition on $C$ is necessary. H. Whithney [4], with J. H. Wolfe, by the introduction of certain norms, has found general abstract spaces of curves along which the integral of 1-forms satisfying certain conditions can be defined. In fact, $H$. Whitney considers integration of $p$-forms with $p \geq 1$. In a previous paper [2], we obtained rather awkward conditions for a decent integral to exist that depended on the number of higher derivatives of $\omega$ on $C$.

In this paper, we consider 1-forms $\omega$ possessing ' higher derivatives' on $C$ in a sense somewhat different from that due to $\mathrm{H}$. Whitney [3] which we used previously. A Lipschitz type condition on the remainders of the Taylor expansion is imposed (see 4.1.). We define the $\alpha$-variation of a curve as the supremum of sums of $\alpha$ th powers of chords (see 2.7) and show that the integral of $\omega$ along $C$ exists if the $\alpha$-variation of $C$ is bounded, where $\alpha$ is related to the number of 'higher derivatives' of $\omega$ on $C$. Under somewhat stronger hypotheses on $C$, we show that this integral is an anti-derivative of $\omega$ on $C$.

2. Notation and basic definitions. Throughout this paper, $N$ is a positive integer and we use the following notation.

2.1. $E$ denotes Euclidean $(N+1)$-space.

2.2. $\|x\|=\left(\sum_{i=0}^{N} x_{i}^{2}\right)^{1 / 2}$ for $x \in E$.

2.3. $\operatorname{diam} U=\sup \{d: d=\|x-y\|$ for some $x \in U$ and $y \in U\}$

2.4. $\varphi$ is a continuous function on the closed unit enterval to $E$ and $C=$ range $\varphi$.

2.5. $\mathscr{S}$ is the set of all subdivisions of the unit interval, i.e. functions $T$ on $\{0,1, \cdots, k\}$ for some positive integer $k$ such that: $T(0)=0, \quad T(k)=1, \quad T(i-1)<T(i)$ for $i=1, \cdots, k$

2.6. $[T / a, b]=\{i: a \leq T(i-1)<T(i) \leq b\}$

2.7. $V_{\alpha}(a, b)=\sup _{T \in \mathscr{S}^{i \in[T / a, b]}} \| \varphi\left(T(i-1)-\varphi(T(i)) \|^{\alpha}\right.$

Received August 25, 1958. 


\section{Properties of $V_{\alpha}$.}

3.1. Lemma. If $0 \leq a \leq b \leq c \leq 1$, then

$$
V_{\alpha}(a, b)+V_{\alpha}(b, c) \leq \alpha(a, c) \leq V_{\alpha}(a, b)+V_{\alpha}(b, c)+(\operatorname{diam} C)^{x}
$$

3.2. Lemma. If $\alpha<\beta$ and $V_{a}(a, b)<\infty$, then $V_{\beta}(a, b)>\infty$.

Proof. Since $V_{\alpha}(a, b)<\infty$, there is an integer $n$ such that there are at most $n$ elements $i \in[T / a, b]$ with $\|\varphi(T(i-1))-\varphi(T(i))\| \geq 1$ for any $T \in \mathscr{S}$. For any other $i \in[T / a, b]$ we have

$$
\|\varphi(T(i-1))-\varphi(T(i))\|^{\beta}<\|\varphi(T(i-1))-\varphi(T(i))\|^{\alpha} .
$$

Hence,

$$
V_{\beta}(a, b)<V_{\alpha}(a, b)+n(\operatorname{diam} C)^{\beta}<\infty .
$$

4. Integration of 1-forms. In this section, we first define the kind of differential form we shall be dealing with. Our definition is a variant of Whitney's definition of a function $m$ times differentiable on a closed set [3]. Next, we choose a special sequence of subdivisions and proceed to define the integral of the form over the curve $C$ by taking sums of polynomials of degree $m$ and then passing to the limit. Under conditions involving the generalized variation $V_{\alpha}$, we show that the integral exists and possesses, in particular, the properties of linearity and 'antiderivative'.

Throughout this section, $m$ is a positive integer, $\eta \geq 0, K>0$.

\subsection{The Differential Form. Let}

$$
\sigma k=\sum_{i=1}^{N} k_{i} \text { for any }(N+1) \text {-tuple } k \text {. }
$$

A differential 1-form $\omega$ on $C$ is a function on the set of all $(N+1)$ tuples $k$, for which $k_{i}$ is a non-negative integer for $i=0, \cdots, N$ and $1 \leq \sigma k \leq m$, to the set of real-valued functions on $C$ such that

$$
\omega_{k}(y)=\sum_{\sigma j=0}^{m-\sigma k} \omega_{k+j}(x) \frac{\left(y_{0}-x_{0}\right)^{j} \cdots\left(y_{N}-x_{N}\right)^{j_{N}}}{j_{0} ! \cdots j_{N} !}+R_{k}(x, y)
$$

where

$$
\left|R_{k}(x, y)\right|<K\|x-y\|^{m+\eta-\sigma k} \text { for } x \in C \text { and } y \in C \text {. }
$$

It is important to note that, in case $m=1$ and $\eta>0, \omega$ is a differential form on $C$ satisfying a Hölder condition. If however $m>1$, then $\omega$ is also a closed differential form on $C$, that is, $d \omega=0$ on $C$. 
By taking $m=1$ and $\eta=1$, we get the sharp forms considered by Whitney. The conditions we impose on $C$, however, are quite different and, we feel, in practice easier to check than those obtained in [4].

4.2. The sequence of subdivisions. We define first, for each $(n+1)$ tuple of non-negative integers $\left(s_{0}, \cdots, s_{n}\right)$, a point $t\left(s_{0}, \cdots, s_{n}\right)$ by recursion on $n$ and on $s_{n}$. These will be the end-points of the $n$th subdivision of the unit interval.

4.2.1. Definition. $t(0)=0, \quad t(1)=1$,

$$
\begin{gathered}
t\left(s_{0}, \cdots, s_{n}, 0\right)=t\left(s_{0}, \cdots, s_{n}\right), \\
t\left(s_{0}, \cdots, s_{n}, j+1\right)=\sup \left\{u: t\left(s_{0}, \cdots, s_{n}, j\right) \leq u \leq t\left(s_{0}, \cdots, s_{n}+1\right)\right.
\end{gathered}
$$

and $\| \varphi\left(u^{\prime}\right)-\varphi\left(t\left(s_{0}, \cdots, s_{n}, j\right) \| \leq \frac{1}{2^{n+1}}\right.$ for $\left.t\left(s_{0}, \cdots, s_{n}, j\right) \leq u^{\prime} \leq u\right\}$

for any non-negative integers $n$ and $j$.

We shall denote by $T$ the sequence of subdivisions of the unit interval such that:

range $T_{n}=\left\{u: u=t\left(s_{0}, \cdots, s_{n}\right)\right.$ for some $n$-tuple $\left.\left(s_{0}, \cdots, s_{n}\right)\right\}$.

4.2.2. Lemma. For any non-negative integers n and $j$, we have

$$
t\left(s_{0}, \cdots, s_{n}\right) \leq t\left(s_{0}, \cdots, s_{n}, j\right) \leq t\left(s_{0}, \cdots, s_{n}+1\right) \text {. }
$$

4.2.3. Lemma. For any positive integer $n, i \in\left[T_{n} / 0,1\right], j \in\left[T_{n-1} / 0,1\right]$ we have: $T_{n+1}$ is a refinement of $T_{n}$, i.e. range $T_{n} \subset$ range $T_{n+1}$;

if

$$
T_{n}(i-1) \leq u \leq T_{n}(i),
$$

then

$$
\left\|\varphi\left(T_{n}(i-1)\right)-\varphi(u)\right\| \leq \frac{1}{2^{n}}
$$

if

$$
T_{n-1}(j-1) \leq T_{n}(i-1)<T_{n}(i)<T_{n-1}(j),
$$

then

$$
\left\|\varphi\left(T_{n}(i-1)\right)-\varphi\left(T_{n}(i)\right)\right\|=\frac{1}{2^{n}} .
$$

4.2.4 Lemma. If $F(x, y)$ is a real number whenever $0 \leq x \leq y \leq 1$, $a \in$ range $T_{n}, b \in$ range $T_{n}$, and $a \leq b$, then $\sum_{i \in\left[T_{n+1} / a, b\right]} F\left(T_{n+1}(i-1), T_{n+1}(i)\right)=\sum_{j \in\left[T_{n^{\prime}} /, b\right]} \sum_{i \in\left[T_{n+1} / T_{n}(j-1), T_{n}(j)\right]} F\left(T_{n+1}(i-1), T_{n+1}(i)\right)$. 
4.3. The integral of $\omega$. First, we define $\int_{b}^{a} \omega d \varphi$ as the limit of certain sums of polynomials.

4.3.1. Definitions.

$$
\begin{aligned}
& P^{\prime}(x, y)=\sum_{\sigma k=1}^{m} \omega_{k}(x) \frac{\left(y_{0}-x_{0}\right)^{k_{0}} \cdots\left(y_{N}-x_{N}\right)^{k_{N}}}{k_{0} ! \cdots k_{N} !}, \\
& P(a, b)=P^{\prime}(\varphi(a), \varphi(b)), \\
& S_{n}(a, b)=\sum_{i \in\left[T_{n}^{\prime} /, b\right]} P\left(T_{n}(i-1), T_{n}(i)\right), \\
& \int_{a}^{b} \omega d \varphi=\lim _{n \rightarrow \infty} S_{n}(a, b) .
\end{aligned}
$$

Next, in order to prove the existence of $\int_{a}^{b} \omega d \varphi$ and some of its properties under conditions involving $V_{a}(a, b)$ for some $\alpha<m+\eta$, we introduce the following.

\subsubsection{Definitions.}

$$
\begin{gathered}
R(x, y, z)=P^{\prime}(x, y)+P^{\prime}(y, z)-P^{\prime}(x, z) . \\
M=K \sum_{\sigma k=1}^{m} \frac{1}{k_{0} ! \cdots k_{N} !} . \\
\beta=m+\eta .
\end{gathered}
$$

4.3.3. Lemma. If $x, y, z \in C,\|x-y\| \leq \delta$ and $\|y-z\| \leq \delta$, then

$$
|R(x, y, z)|<M \delta^{3} \text {. }
$$

Proof. Let $h(v)=P^{\prime}(x, v)$ for $v \in E$. Then, $h$ is a polynomial of degree $m$. Let $\boldsymbol{O}_{r}=\{k: k$ is an $(N+1)$-tuple of non-negative integers and $1 \leq \sigma k \leq r\}$.

For $k \in \boldsymbol{O}_{r}$ and $p \in \boldsymbol{O}_{r}$, let $p \geq k$ iff $p_{i} \geq k_{i}$ for $i=0, \cdots, N$, and let

$$
D_{k} h(v)=\frac{\partial^{\sigma k} h(v)}{\partial^{k} v_{0} \cdots \partial^{k} v_{N}},
$$

then

$$
D_{k} h(v)=\sum_{\substack{p \in \boldsymbol{o}_{m} \\ p \geq k}} \omega_{p}(x) \frac{\left(v_{0}-x_{0}\right)^{p_{0}}-k_{0} \cdots\left(v_{N}-x_{N}\right)^{p_{N} k_{N}}}{\left(p_{0}-k_{0}\right) ! \cdots\left(p_{N}-k_{N}\right) !} .
$$

Hence, by Taylor's formula

$$
h(z)=h(y)+\sum_{k \in \boldsymbol{O}_{m}} D_{k} h(y) \frac{\left(z_{0}-y_{0}\right)^{k_{0}} \cdots\left(z_{N}-y_{N}\right)^{k_{N}}}{k_{0} ! \cdots k_{N} !}=h(y)+
$$




$$
\begin{array}{r}
+\sum_{k \in \boldsymbol{O}_{m}}\left\{\left[\sum_{\substack{p \in \boldsymbol{O}_{m} \\
p \geq k}} \omega_{p}(x) \frac{\left(y_{0}-x_{0}\right)^{p_{0}-k_{0}} \cdots\left(y_{N}-x_{N}\right)^{p}{ }^{-k_{N}}}{\left(p_{0}-k_{0}\right) ! \cdots\left(p_{N}-k_{N}\right) !}\right]\right. \\
\left.\cdot \frac{\left(z_{0}-y_{0}\right)^{k_{0}} \cdots\left(z_{N}-y_{N}\right)^{k}}{k_{0} ! \cdots k_{N} !}\right\} .
\end{array}
$$

On the other hand from 4.3.1 and 4.1 we have

$$
\begin{aligned}
& P^{\prime}(y, z)=\sum_{k \in \boldsymbol{o}_{m}}\left\{\left[\omega_{k}(x)+\sum_{j \in \boldsymbol{o}_{m-\sigma k}} \omega_{k+j}(x) \frac{\left(y_{0}-x_{0}\right)^{j_{0}} \cdots\left(y_{N}-x_{N}\right)^{j_{N}}}{j_{0} ! \cdots j_{N} !}+R_{k}(x, y)\right]\right. \\
& \left.\frac{\left(z_{0}-y_{0}\right)^{k_{0}} \cdots\left(z_{N}-y_{N}\right)^{k_{N}}}{k_{0} ! \cdots k_{N} !}\right\} \\
& =\sum_{k \in \boldsymbol{O}_{m}}\left\{\left[\sum_{\substack{k \in \boldsymbol{O}_{m} \\
p \geq k}} \omega_{p}(x) \frac{\left(y_{0}-x_{0}\right)^{p_{0}}-k_{0} \cdots\left(y_{N}-x_{N}\right)^{p_{N}-k_{N}}}{\left(p_{0}-k_{0}\right) ! \cdots\left(p_{N}-k_{N}\right) !}+R_{k}(x, y)\right]\right. \\
& \left.\left.\frac{\left(z_{0}-x_{0}\right)^{k_{0}} \cdots\left(z_{N}-y_{N}\right)^{k_{N}}}{k_{0} ! \cdots k_{N} !}\right]\right\} \\
& =h(z)-h(y)+\sum_{k \in \boldsymbol{o}_{m}} R_{k}(x, y) \frac{\left(z_{0}-y_{0}\right)^{k_{0}} \cdots\left(z_{N}-y_{N}\right)^{k_{N}}}{k_{0} ! \cdots k_{N} !} .
\end{aligned}
$$

Making use of the condition on $R_{k}(x, y)$ stated in 4.1 , we get $\left|P^{\prime}(x, y)+P^{\prime}(y, z)-P^{\prime}(x, z)\right|<\sum_{k \in \boldsymbol{o}_{m}} \frac{K\|y-x\|^{\beta-\sigma k}\|z-y\|^{\sigma k}}{k_{0} ! \cdots k_{N} !} \leq M \delta^{\beta}$.

4.3.4 Lemma. Suppose $\|x(0)-x(i)\| \leq A$ and $\|x(i-1)-x(i)\| \leq$ $A$ for $i=1, \cdots, p$, whereas $\|x(i-1)-x(i)\|=A / r$ for $i=1, \cdots, p-1$, where all $x(i) \in C$. Then

$$
\left|\sum_{i=1}^{p} P^{\prime}(x(i-1), x(i))-P^{\prime}(x(0), x(p))\right|<M r^{\alpha} A^{\beta-\alpha} \sum_{i=1}^{p}\|x(i-1)-x(i)\|^{\alpha} .
$$

$$
\begin{aligned}
\text { Proof. } & \left|\sum_{i=1}^{p} P^{\prime}(x(i-1), x(i))-P^{\prime}(x(0), x(p))\right| \\
& \leq \sum_{i=2}^{p}\left|P^{\prime}(x(0), x(i-1))+P^{\prime}(x(i-1), x(i))-P^{\prime}(x(0), x(i))\right| \\
& =\sum_{i=2}^{p-1}|R(x(0), x(i-1), x(i))|<(p-1) M A^{\beta}=(p-1) M r^{x} A^{\beta-\alpha}\left(\frac{A}{r}\right)^{\alpha} \\
& =M r^{\alpha} A^{\beta-\alpha} \sum_{i=1}^{p-1}\|x(i-1)-x(i)\|^{\alpha} \leq M r^{\alpha} A^{\beta-\alpha} \sum_{i=1}^{p}\|x(i-1)-x(i)\|^{\alpha} .
\end{aligned}
$$

4.3.5 Lemma. Let $n>1, a \in$ range $T_{n}, b \in$ range $T_{n}, a \leq b$,

$$
\begin{aligned}
& {\left[T_{n-1} / a, b\right]=0 . \text { Then }} \\
& \left|S_{n}(a, b)-P(a, b)\right|<M 5^{\beta} V_{\beta}(a, b) .
\end{aligned}
$$

Proof. Let

$$
\begin{aligned}
& a^{\prime}=\sup \left\{u: u \in \text { range } T_{n-1} \text { and } u \leq a\right\} \\
& b^{\prime}=\sup \left\{u: u \in \operatorname{range} T_{n-1} \text { and } u \leq b\right\} .
\end{aligned}
$$


First, suppose $a \leq b^{\prime} \leq b$. Then $a^{\prime}<a$ and, by 4.2 .3

$$
\begin{array}{ll}
\left\|\varphi(u)-\varphi\left(a^{\prime}\right)\right\| \leq \frac{1}{2^{n-1}} & \text { for } a^{\prime} \leq u \leq b^{\prime} \\
\left\|\varphi(u)-\varphi\left(b^{\prime}\right)\right\| \leq \frac{1}{2^{n-1}} & \text { for } b^{\prime} \leq u \leq b .
\end{array}
$$

Hence

$$
\begin{aligned}
& \left\|\varphi\left(T_{n}(i)\right)-\varphi(a)\right\| \leq-\frac{2}{2^{n-1}} \quad \text { for } i \in\left[T_{n} / a, b\right], \\
& \left\|\varphi\left(T_{n}(i)\right)-\varphi\left(b^{\prime}\right)\right\| \leq \frac{1}{2^{n-1}} \quad \text { for } i \in\left[T_{n} \mid b^{\prime}, b\right],
\end{aligned}
$$

$\left\|\varphi\left(T_{n}(i-1)\right)-\varphi\left(T_{n}(i)\right)\right\|=\frac{1}{2^{n}} \quad$ for $i \in\left[T_{n} / a, b\right], T_{n}(i) \neq b^{\prime}, T_{n}(i) \neq b$.

Replacing $\alpha$ by $\beta$ in 4.3.4 and using 4.3.3 and 3.1, we see that

$$
\begin{aligned}
& \left|S_{n}(a, b)-P(a, b)\right|=\left|S_{n}\left(a, b^{\prime}\right)+S_{n}\left(b^{\prime}, b\right)-P(a, b)\right| \\
\leq & \left|S_{n}\left(a, b^{\prime}\right)-P\left(a, b^{\prime}\right)\right|+\left|S_{n}\left(b^{\prime}, b\right)-P\left(b^{\prime}, b\right)\right|+\left|P\left(a, b^{\prime}\right)+P\left(b^{\prime}, b\right)-P(a, b)\right| \\
< & M 4^{\beta} V_{\beta}\left(a, b^{\prime}\right)+M 2^{\beta} V_{\beta}\left(b^{\prime}, b\right)+M V_{\beta}(a, b) \leq M 5^{\beta} V_{\beta}(a, b) .
\end{aligned}
$$

Next suppose $b^{\prime}<a$. Then, for $i \in\left[T_{n} / a, b\right]$,

$$
\begin{gathered}
\left\|\varphi\left(T_{n}(i)\right)-\varphi(a)\right\| \leq \frac{2}{2^{n-1}}, \\
\left\|\varphi\left(T_{n}(i-1)\right)-\varphi\left(T_{n}(i)\right)\right\|=\frac{1}{2^{n}} .
\end{gathered}
$$

Hence, by 4.3.4,

$$
\left|S_{n}(a, b)-P(a, b)\right|<M 4^{\beta} V_{\beta}(a, b) .
$$

4.3.6 Lemma. Let $a \in$ range $T_{n}, b \in$ range $T_{n}, a<b$. Then,

$$
\left|S_{n+1}(a, b)-S_{n}(a, b)\right|<M 2^{\alpha} V_{\alpha}(a, b)\left(\frac{1}{2^{\beta-\alpha}}\right)^{n} .
$$

Proof. Using 4.2.4, 4.2.3 and 4.3.4, we see that

$$
\left|S_{n+1}(a, b)-S_{n}(a, b)\right|
$$$$
=\left|\sum_{j \in\left[T_{T^{\prime}} \mid a, b\right]}\left[\sum_{i \in\left[T_{n+1} / T_{n}^{\left.(j-1), T_{n}(j)\right]}\right.} P\left(T_{n+1}(i-1), T_{n+1}(i)\right)-P\left(T_{n}(j-1), T_{n}(j)\right)\right]\right|
$$$$
<\sum_{j \in\left[T_{n} / a, b\right]}\left[M 2^{\alpha}\left(\frac{1}{2^{n}}\right)^{\beta-\alpha} \sum_{t \in\left[T_{n+1} / T_{n}^{\left.(j-1), T_{n}(j)\right]}\right.}\left\|\varphi\left(T_{n+1}(i-)\right)-\varphi\left(T_{n+1}(i)\right)\right\|^{\alpha}\right]
$$$$
=M 2^{\alpha}\left(\frac{1}{2^{n}}\right)^{\beta-\alpha} \sum_{i \in\left[T_{n+1} / a, b\right]}\left\|\varphi\left(T_{n+1}(i-1)\right)-\varphi\left(T_{n+1}(i)\right)\right\|^{\alpha} \leq M 2^{\alpha} V_{\alpha}(a, b)\left(\frac{1}{2^{\beta-\alpha}}\right)^{n} .
$$

4.3.7. Theorem. If $0 \leq a \leq b \leq 1, \alpha<\beta, V_{a}(a, b)<\infty$, then 


$$
\left|\int_{n}^{b} \omega d \varphi\right|<\infty
$$

Proof. Let

$$
\begin{aligned}
& a_{n}^{\prime}=\inf \left\{u: u \in \operatorname{range} T_{n} \text { and } a \leq u\right\}, \\
& b_{n}^{\prime}=\sup \left\{u: u \in \operatorname{range} T_{n} \text { and } u \leq b\right\} .
\end{aligned}
$$

If $a=b$, the theorem is trivial. If $a<b$, for $n$ sufficiently large, we have

$$
\begin{gathered}
a \leq a_{n+1}^{\prime} \leq a_{n}^{\prime} \leq b_{n}^{\prime} \leq b_{n+1}^{\prime} \leq b, \\
{\left[T_{n} / a, a_{n}^{\prime}\right]=0 \quad \text { and }\left[T_{n} / b_{n}^{\prime}, b\right]=0,} \\
\left\|\varphi\left(a_{n+1}^{\prime}\right) \varphi-\left(a_{n}^{\prime}\right)\right\| \leq \frac{2}{2^{n}} \quad \text { and }\left\|\varphi\left(b_{n}^{\prime}\right)-\varphi\left(b_{n+1}^{\prime}\right)\right\| \leq \frac{1}{2^{n}} .
\end{gathered}
$$

Hence

$$
\begin{gathered}
\left|S_{n+1}(a, b)-S_{n}(a, b)\right|=\left|S_{n+1}\left(a_{n+1}^{\prime}, b_{n+1}^{\prime}\right)-S_{n}\left(a_{n}^{\prime}, b_{n}^{\prime}\right)\right| \\
=\left|S_{n+1}\left(a_{n+1}^{\prime}, a_{n}^{\prime}\right)+S_{n+1}\left(a_{n}^{\prime}, b_{n}^{\prime}\right)+S_{n+1}\left(b_{n}^{\prime}, b_{n+1}^{\prime}\right)-S_{n}\left(a_{n}^{\prime}, b_{n}^{\prime}\right)\right| \\
\leq\left|S_{n+1}\left(a_{n+1}^{\prime}, a_{n}^{\prime}\right)-P\left(a_{n+1}^{\prime}, a_{n}^{\prime}\right)\right|+\left|S_{n+1}\left(a_{n}^{\prime}, b_{n}^{\prime}\right)-S_{n}\left(a_{n}^{\prime}, b_{n}^{\prime}\right)\right| \\
+\left|S_{n+1}\left(b_{n}^{\prime}, b_{n+1}^{\prime}\right)\right|-P\left(b_{n}^{\prime}, b_{n+1}^{\prime}\right)|+| P\left(a_{n+1}^{\prime}, a_{n}^{\prime}\right) \mid+1 P\left(b_{n}^{\prime}, b_{n+1}^{\prime} \mid<(\text { by 4.3.5, 4.3.6) }\right.
\end{gathered}
$$$$
<M 5^{\beta} V_{\beta}\left(a_{n+1}^{\prime}, a_{n}^{\prime}\right)+M 2^{x} V_{\alpha}\left(a_{n}^{\prime}, b_{n}^{\prime}\right)\left(\frac{1}{2^{\beta-\alpha}}\right)^{n}+M 5^{\beta} V_{\beta}\left(b_{n}^{\prime}, b_{n+1}^{\prime}\right)+M^{\prime} \cdot \frac{2}{2^{n}}+M^{\prime} \frac{1}{2^{n}} \text {, }
$$

where

$$
M^{\prime}=\sup _{\substack{x \in \sigma \\ 1 \leq \sigma k<m}}\left|\omega_{k}(x)\right| \sum_{\sigma k=1}^{m} \frac{1}{k_{0} ! \cdots k_{N} !} .
$$

Therefore, for any positive integer $p$ we have

$$
\begin{gathered}
\left|S_{n+p}(a, b)-S_{n}(a, b)\right| \leq \sum_{q=0}^{p-1}\left|S_{n+q+1}(a, b)-S_{n+q}(a, b)\right| \\
<M 5^{\beta} \sum_{q=0}^{\infty}\left[V_{\beta}\left(a_{n+q+1}^{\prime}, a_{n+q}^{\prime}\right)+V_{\beta}\left(b_{n+q}^{\prime}, b_{n+n+q+1}^{\prime}\right)\right]+M 2^{x} V_{\alpha}(a, b) \sum_{q=0}^{\infty}\left(\frac{1}{2^{\beta-\alpha}}\right)^{n+q} \\
+3 M^{\prime} \sum_{q=0}^{\infty} \frac{1}{2^{n+q}}<M 5^{\beta}\left(V_{\beta}\left(a, a_{n}^{\prime}\right)+V_{\beta}\left(b_{n}^{\prime}, b\right)\right)+M \frac{2^{3}}{2^{3-\alpha}-1} V_{\alpha}(a, b)\left(\frac{1}{2^{3-\alpha}}\right)^{n}+\frac{6 M^{\prime}}{2^{n}} .
\end{gathered}
$$

Since, by 3.2, $V_{\beta}(a, b)<\infty$, with the help of 3.1 we see that $V_{\beta}\left(a, a_{n}^{\prime}\right) \rightarrow 0$ and $V_{\beta}\left(b_{n}^{\prime}, b\right) \rightarrow 0$ as $n \rightarrow \infty$. Thus, the $S_{n}(a, b)$ form a Cauchy sequence and $\left|\int_{a}^{b} \omega d \varphi\right|<\infty$.

4.3.8. Theorem. Suppose $\delta>0, \alpha<\beta, L<\infty,\|\varphi(a)-\varphi(b)\|<1$, and

$$
V_{\alpha}(a, b)<L\|\varphi(a)-\varphi(b)\|^{\alpha}
$$

whenever $0 \leq a \leq b \leq 1$ and $b-a<\delta$. Then, for some $M^{\prime}<\infty$, 


$$
\left|\int_{a}^{b} \omega d \varphi-P(a, b)\right|<M^{\prime}\|\varphi(a)-\varphi(b)\|^{\alpha}
$$

whenever $0 \leq a \leq b \leq 1$ and $b-a<\delta$.

Proof. Given $0 \leq a \leq b \leq 1$ and $b-a<\delta$, let

$$
\begin{aligned}
& a_{q}^{\prime}=\inf \left\{u: u \in \operatorname{range} T_{q} \text { and } a \leq u\right\}, \\
& b_{q}^{\prime}=\sup \left\{u: u \in \operatorname{range} T_{q} \text { and } u \leq b\right\} ;
\end{aligned}
$$

and let $n$ be the integer such that $\left[T_{n-1} / a, b\right]=0,\left[T_{n} / a, b\right] \neq 0$.

Given $\varepsilon>0$, we can choose $p$ so that

$$
\left|\int_{a}^{b} \omega d \varphi-S_{n+p}\left(a_{n+p}^{\prime}, b_{n+p}^{\prime}\right)\right|<\varepsilon
$$

and

$$
\left|P(a, b)-P\left(\begin{array}{l}
\prime \\
n+p
\end{array}, b_{n+p}^{\prime}\right)\right|<\varepsilon
$$

and

$$
\left|\|\varphi(a)-\varphi(b)\|-\left\|\varphi\left(a_{n+p}^{\prime}\right)-\varphi\left(b_{n+p}^{\prime}\right)\right\|\right|<\varepsilon .
$$

Hence we need only to show that

$$
\left|S_{n+p}\left(a_{n+p}^{\prime}, b_{n+p}^{\prime}\right)-P\left(a_{n+p}^{\prime}, b_{n+p}^{\prime}\right)\right|<M^{\prime}\left\|\varphi\left(a_{n+p}^{\prime}\right)-\varphi\left(b_{n+p}^{\prime}\right)\right\|^{\alpha}
$$

for some $M^{\prime}<\infty$ and all positive integers $p$.

We can check that

$$
\begin{aligned}
& \left|S_{n+p}\left(a_{n+p}^{\prime}, b_{n+p}^{\prime}\right)-P\left(a_{n+p}^{\prime}, b_{n+p}^{\prime}\right)\right| \\
\leq & \left|S_{n}\left(a_{n}^{\prime}, b_{n}^{\prime}\right)-P\left(a_{n}^{\prime}, b_{n}^{\prime}\right)\right|+\left|P\left(a_{n+p}^{\prime}, a_{n}^{\prime}\right)+P\left(a_{n}^{\prime}, b_{n}^{\prime}\right)-P\left(a_{n+p}^{\prime}, b_{n}^{\prime}\right)\right| \\
+ & \left|P\left(a_{n+p}^{\prime}, b_{n}^{\prime}\right)+P\left(b_{n}^{\prime}, b_{n+p}^{\prime}\right)-P\left(a_{n+p}^{\prime}, b_{n+p}^{\prime}\right)\right| \\
+ & \sum_{k=0}^{p-1}\left\{\mid P\left(a_{n+p}^{\prime}, a_{n+k+1}^{\prime}\right)+P\left(\left(a_{n+k+1}^{\prime}, a_{n+k}^{\prime}\right)-P\left(a_{n+p}^{\prime}, a_{n+k}^{\prime}\right) \mid\right.\right. \\
+ & \left|P\left(b_{n+k}^{\prime}, b_{n+k+1}^{\prime}\right)+P\left(b_{n+k+1}^{\prime}, b_{n+p}^{\prime}\right)-P\left(b_{n+k}^{\prime}, b_{n+p}^{\prime}\right)\right| \\
+ & \left|S_{n+k+1}\left(a_{n+k+1}^{\prime}, a_{n+k}^{\prime}\right)-P\left(a_{n+k+1}^{\prime}, a_{n+k}^{\prime}\right)\right| \\
+ & \left|S_{n+k+1}\left(b_{n+k}^{\prime}, b_{n+k+1}^{\prime}\right)-P\left(b_{n+k+1}^{\prime}, b_{n+k}^{\prime}\right)\right| \\
+ & \left.\left|S_{n+k+1}^{\prime}\left(a_{n+k}^{\prime}, b_{n+k}^{\prime}\right)-S_{n+k}\left(a_{n+k}^{\prime}, b_{n+k}^{\prime}\right)\right|\right\} .
\end{aligned}
$$

Now, we observe that

$$
\begin{gathered}
\|\varphi(u)-\varphi(v)\| \leq \frac{2}{2^{n+k}} \quad \text { for } a_{n+p}^{\prime} \leq u \leq v \leq a_{n+k}^{\prime}, \\
\|\varphi(u)-\varphi(v)\| \leq \frac{1}{2^{n+k}} \quad \text { for } b_{n+k}^{\prime} \leq u \leq v \leq b_{n+p}^{\prime}, \\
{\left[T_{n+k} / a_{n+k+1}^{\prime}, a_{n+k}^{\prime}\right]=0,} \\
{\left[T_{n+k} / b_{n+k}^{\prime}, b_{n+k+1}^{\prime}\right]=0 .}
\end{gathered}
$$

Hence by $4.3 .5,4.3 .3,4.3 .6$ we have 


$$
\begin{aligned}
& \left|S_{n+p}^{\prime}\left(a_{n+p}^{\prime}, b_{n+p}^{\prime}\right)-P\left(a_{n+p}^{\prime}, b_{n+p}^{\prime}\right)\right| \\
< & M 5^{\beta} V_{\beta}\left(a_{n}^{\prime}, b_{n}^{\prime}\right)+M V_{\beta}\left(a_{n+p}^{\prime}, b_{n}^{\prime}\right)+M V_{\beta}\left(a_{n+p}^{\prime}, b_{n+p}^{\prime}\right) \\
+ & M \sum_{k=0}^{p-1}\left\{V_{\alpha}\left(a_{n+p}^{\prime}, a_{n+k}^{\prime}\right)\left(\frac{2}{2^{n+k}}\right)^{\beta-\alpha}+V_{\alpha}\left(b_{n+k}^{\prime}, b_{n+p}^{\prime}\right)\left(\frac{1}{2^{n+k}}\right)^{\beta-\alpha}\right. \\
+ & \left.5^{\beta} V_{\beta}\left(a_{n+k+1}^{\prime}, a_{n+k}^{\prime}\right)+5^{\beta} V_{\beta}\left(b_{n+k}^{\prime}, b_{n+k+1}^{\prime}\right)+2^{\alpha} V_{\alpha}\left(a_{n+k}^{\prime}, b_{n+k}^{\prime}\right)\left(\frac{1}{2^{\beta-\alpha}}\right)^{n+k}\right\} \\
< & M 5^{\beta} V_{\beta}\left(a_{n+p}^{\prime}, b_{n+p}^{\prime}\right)+2 M V_{\beta}\left(a_{n+p}^{\prime}, b_{n+p}^{\prime}\right) \\
+ & M V_{\alpha}\left(a_{n+p}^{\prime}, b_{n+p}^{\prime}\right)\left(2^{\beta-\alpha}+1+2^{x}\right) \sum_{k=0}^{\infty}\left(\frac{1}{2^{\beta-\alpha}}\right)^{n+k} \\
< & M V_{\alpha}\left(a_{n+p}^{\prime}, b_{n+p}^{\prime}\right)\left[5^{\beta}+2+\left(2^{\beta-\alpha}+1+2^{\alpha}\right) \sum_{k=0}^{\infty}\left(\frac{1}{2^{\beta-\alpha}}\right)^{n+k}\right] \\
< & M\left\|\varphi\left(a_{n+p}^{\prime}\right)-\varphi\left(b_{n+p}^{\prime}\right)\right\|^{\alpha}
\end{aligned}
$$

where

$$
M^{\prime}=M L\left[5^{\beta}+2+\left(2^{3-\alpha}+1+2^{x}\right) \sum_{k=0}^{\infty}\left(\frac{1}{2^{\beta-\alpha}}\right)^{n+k}\right]<\infty .
$$

4.3.9. THEOREM. If $0 \leq a \leq b \leq c \leq 1,\left|\int_{a}^{b} \omega d \varphi+\int_{b}^{c} \omega d \varphi\right|<\infty$, then

$$
\int_{a}^{c} \omega d \varphi=\int_{a}^{b} \omega d \varphi+\int_{b}^{c} \omega d \varphi
$$

Proof. Let

$$
\begin{aligned}
& a_{n}^{\prime}=\sup \left\{u: u \in \operatorname{range} T_{n} \text { and } u \leq b\right\} \\
& b_{n}^{\prime}=\inf \left\{u: u \in \operatorname{range} T_{n} \text { and } b \leq u\right\} .
\end{aligned}
$$

We have $\lim _{n \rightarrow \infty} P\left(a_{n}^{\prime}, b_{n}^{\prime}\right)=0$ and for sufficiently large $n$

$$
S_{n}(a, c)=S_{n}(a, b)+P\left(a_{n}^{\prime}, b_{n}^{\prime}\right)+S_{n}(b, c) .
$$

Taking the limit on both sides we get the desired result.

4.3.10. REMARK. If $\omega$ and $\omega^{\prime}$ are both 1-forms in the sense of 4.1, then so is $\left(\omega+\omega^{\prime}\right)$ and

$$
\int_{a}^{b}\left(\omega+\omega^{\prime}\right) d \varphi=\int_{a}^{b} \omega d \varphi+\int_{a}^{b} \omega^{\prime} d \varphi
$$

provided the right hand side is bounded. This is an immediate consequence of the definitions.

\section{REFERENCES}

1. G. Glaeser, Etudes de quelques algèbres tayloriennes, J. d'Analyse Math., Jerusalem, 6, (1958), 1-124. 
2. M. Sion, On the existence of functions having given partial derivatives on a curve, Trans. Am. Math. Soc. 77 (1954), 179-201.

3. H. Whitney, Analytic extensions of differentiable functions defined on closed sets, Trans. Am. Math. Soc. 36 (1934), 63-89.

4. — Geometric integration theory, Princeton Univ. Press, 1957.

UNIVERSITY OF CALIFORNIA 


\section{PACIFIC JOURNAL OF MATHEMATICS}

\section{EDITORS}

\section{David Gilbarg}

Stanford University

Stanford, California

R. A. Beaumont

University of Washington

Seattle 5 , Washington
A. L. Whiteman

University of Southern California

Los Angeles 7, California

L. J. PAIGE

University of California

Los Angeles 24, California

\section{ASSOCIATE EDITORS}
E. F. BECKENBACH
C. E. BURGESS
E. HEWITT
A. HORN

\author{
V. GANAPATHY IYER \\ R. D. JAMES \\ M. S. KNEBELMAN \\ L. NACHBIN
}
I. NIVEN
E. G. STRAUS
T. G. OSTROM
H. L. ROYDEN
G. SZEKERES
M. M. SCHIFFER
F. WOLF
K. YOSIDA

\section{SUPPORTING INSTITUTIONS}

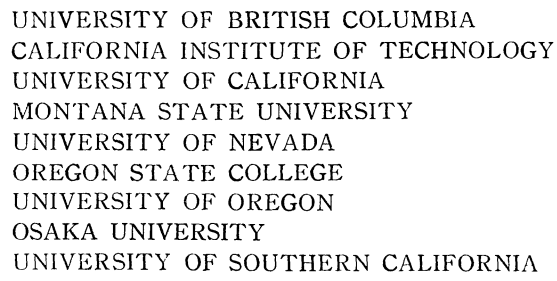

UNIVERSITY OF BRITISH COLUMBIA CALIFORNIA INSTITUTE OF TECHNOLOGY UNIVERSITY OF CALIFORNIA MONTANA STATE UNIVERSITY UNIVERSITY OF NEVADA OREGON STATE COLLEGE UNIVERSITY OF OREGON OSAKA UNIVERSITY UNIVERSITY OF SOUTHERN CALIFORNIA

STANFORD UNIVERSITY

UNIVERSITY OF TOKYO

UNIVERSITY OF UTAH

WASHINGTON STATE COLLEGE

UNIVERSITY OF WASHINGTON

AMERICAN MATHEMATICAL SOCIETY CALIFORNIA RESEARCH CORPORATION HUGHES AIRCRAFT COMPANY SPACE TECHNOLOGY LABORATORIES

Printed in Japan by Kokusai Bunken Insatsusha (International Academic Printing Co., Ltd.), Tokyo, Japan 


\section{Pacific Journal of Mathematics}

\section{Vol. 9, No. 1 \\ May, 1959}

Julius Rubin Blum and Murray Rosenblatt, On the structure of infinitely

divisible distributions . ............................. 1

Robert Geroge Buschman, Asymptotic expressions for

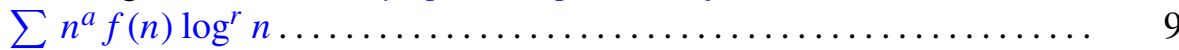

Eckford Cohen, A class of residue systems $(\bmod r)$ and related arithmetical

functions. I. A generalization of Möbius inversion .............. 13

Paul F. Conrad, Non-abelian ordered groups ................... 25

Richard Henry Crowell, On the van Kampen theorem............... 43

Irving Leonard Glicksberg, Convolution semigroups of measures ........ 51

Seymour Goldberg, Linear operators and their conjugates ............ 69

Olof Hanner, Mean play of sums of positional games .............. 81

Erhard Heinz, On one-to-one harmonic mappings ................ 101

John Rolfe Isbell, On finite-dimensional uniform spaces . . ........... 107

Erwin Kreyszig and John Todd, On the radius of univalence of the function

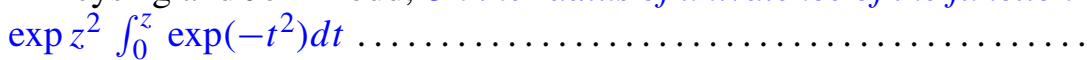

Roger Conant Lyndon, An interpolation theorem in the predicate

calculus......................................... 129

Roger Conant Lyndon, Properties preserved under homomorphism ........ 143

Roger Conant Lyndon, Properties preserved in subdirect products ....... 155

Robert Osserman, A lemma on analytic curves ................ 165

R. S. Phillips, On a theorem due to Sz.-Nagy..................... 169

Richard Scott Pierce, A generalization of atomic Boolean algebras ....... 175

J. B. Roberts, Analytic continuation of meromorphic functions in valued fields................................. 183

Walter Rudin, Idempotent measures on Abelian groups ................ 195

M. Schiffer, Fredholm eigen values of multiply-connected domains ........ 211

V. N. Singh, A note on the computation of Alder's polynomials ......... 271

Maurice Sion, On integration of 1-forms ...................... 277

Elbert A. Walker, Subdirect sums and infinite Abelian groups........... 287

John W. Woll, Homogeneous stochastic processes . .................. 293 